Il me parait intéressant de relever que la maladie frappa d'abord des animaux récemment introduits dans le troupeau et provenant du Brakna (Mauritanie). Est-ce à dire que le virus fut transporté par eux? Je ne le pense pas. La fièvre du tollé est, je le répète, habituelle dans la région saint-louisienne; et il est plus vraisemblable d'admettre que les veaux de race maure, affaiblis par un long parcours et non acclimatés, offraient un terrain éminemment favorable à l'action de virus.

La contagion se fit apparemment par contact, ni tiques, ni poux n'ayant été observés sur les malades. Ie rôle contagifère des petits ruminants parait devoir être écarté, le propriétaire du troupeau atteint n'élevant ni mouton, ni chèvre. Quant aux antilopinës, vecteurs possibles, ils sont absents de la région.

La contamination par l'inoculation de sang d'un animal malade à un animal sain n'a pas été tentée.

On a vu que la tentative faite à Kiffa par SID M Mahamane n'avait pas donné de résultat positif. Ajoutons que les auteurs s'accordent pour reconnaitre qu'il est nécessaire d'injecter une grande quantité de sang pour réaliser la contamination.

J'aurai l'occasion de revenir sur une affection dont l'endémicité est maintenant avérée et qui pourra faire l'objet de nouvelles observations.

\title{
IMMUNITE CONGENITALE
}

\section{ET VIRUS PESTE BOVINE ADAPTE SUR CHEVRE} par J. GILLAIN

Il est de notion courante qu'une vache ayant été immunisée, soịt par infection naturelle, soit par séro-infection, donne naissance à des veaux chez lesquels l'infection naturelle ou la séro-infection, pratiquement inopérantes dans les premiers mois de la vie, n'engendrent sûrement i'état réfractaire qu'à partir de l'âge de six mois, entre six mois et un an selon le cas.

Les veaux de pareilles vaches possèdent donc une immunité congénitale empêchante, mais transitoire. En effet, ces veaux sont doués d'une résistance naturelle d'abord totale qui s'affaiblit ensuite plus ou moins rapidement jusqu'à disparaître complètement. H. Jacotot et M. CoLson (1) avec d'autres la considèrent comme le résultat du fait que les veaux consomment exclusivement d'abord, puis en quantités décroissantes, le lait de leur mère chargé d'anticorps pestiques possédant, comme le sang, des propriétés protectrices. 
L'adaptation du virus peste bovine sur la chèvre n'apporte aucune modification aux phénomènes d'immunité congénitale, ainsi que le montre le tahleau ci-après.

Dans nos expériences, les vaches ont été vaccinées avec le virus vacein constitué par une souche de peste bovine adaptée sur chèvre. Divers modes de préparalion ont élé employéś avec les mêmes résultats : sang citraté de chèvre infectée à la dose de 1 ou 2 cc.; émulsion de rate fraîche de $1 \%$ dans sérum physiologique à 4 pour 1.000, dose 1 ou 2 cc.; émulsion de rate sèche à 1 pour 400 en sérum physiologique à 4 pour 1.000 , dose 1 ou $2 \mathrm{cc}$.

Pour l'épreuve des veaux, le même virus vaccin a été employé.

\begin{tabular}{|c|c|c|c|c|c|c|c|}
\hline $\begin{array}{c}\text { No } \\
\text { VEAU }\end{array}$ & $\begin{array}{c}\text { DATE } \\
\text { NAISSANOE }\end{array}$ & $\begin{array}{c}\text { No } \\
\text { MERTE }\end{array}$ & $\begin{array}{c}\text { DATE } \\
\text { vaccinatiou meres }\end{array}$ & $\begin{array}{c}\text { DATE } \\
\text { épreulre vcalt }\end{array}$ & $\begin{array}{l}\text { ÉTAT } \\
\text { DU VEAU }\end{array}$ & RÉSULTAT & OBSERVATIONS \\
\hline 4889 & $17-10-42$ & 3481 & $20-10-42$ & $26-5-43$ & non sevré & réceptif & \\
\hline $4881^{\prime}$ & $19-10-42$ & $\mid$ & $20-10-42$ & $20-50$ & - & & \\
\hline 4882 & $19-10-42$ & 3090 & - & - & 一 & - & \\
\hline 4895 & $30-10-42$ & 3047 & - & 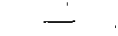 & - & — & \\
\hline 4936 & $15-11-42$ & 2742 & - & 一 & - & immun. & \\
\hline 5022 & $8-1-43$ & 2309 & $\therefore$ & - & - & - & \\
\hline 5050 & $23-2-43$ & 3000 & - & $\therefore$ & - & - & \\
\hline 5046 & $25-2-43$ & 2762 & - & — & 一 & - & \\
\hline 5098 & $17-3-43$ & 2691 & 一 & - & - & - & \\
\hline $\mathbf{5 1 0 3}$ & $29-3-43$ & 3548 & - & - & - & 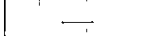 & \\
\hline 5172 & $2-4-43$ & 2582 & - & - & $\cdots$ & -1 & \\
\hline 5165 & $6-4-43$ & 3107 & - & - & 一 & - & \\
\hline 5164 & $19-4-43$ & 2597 & $\div$ & - & - & - & \\
\hline 4885 & $20-10-42$ & 3376 & - & $31-3-44$ & sevré & réceptif & \\
\hline 4886 & $19-10-42$ & 3252 & - & $\therefore \quad$ & - & - & \\
\hline 4954 & $3-11-42$ & $266 \overline{6}$ & - & - & - & - & \\
\hline 5048 & $5-2-43$ & 3186 & - & - & 一 & immun. & \\
\hline 5100 & $26-3-43$ & 3411 & - & 一 & - & - & \\
\hline 5110 & $28-3-13$ & 3683 & - & - & 一 & réceptif & \\
\hline 5111 & $25-3-43$ & 3432 & $\ldots$ & - & 一 & - & \\
\hline 5112 & $28-3-43$ & 3498 & - & — & - & - & \\
\hline 5120 & $26-3-43$ & 3635 & - & $\ldots$ & 一 & - & . \\
\hline 5163 & $8-4-43$ & 3583 & - & - & - & - & \\
\hline 5166 & $20-4-43$ & 3216 & - & - & - & - & \\
\hline 5167 & $27-4-43$ & 3630 & 一 & - & - & - & \\
\hline 5463 & $10-11-43$ & 2788 & - & $8 \cdot 6 \cdot 44$ & All. Art. & - & All. Art: : veau \\
\hline 5736 & $29-4-44$ & 3582 & - & - & - & immun. & séparé de sà \\
\hline 5648 & $20-3-44$ & 3186 & 一 & - & $\ldots$ & $\ldots$ & mêrè à la nais \\
\hline 5506 & $16-12-43$ & 3386 & — & $\ldots$ & non sevré & 一 & alimenté avec \\
\hline 5507 & $27-12 \cdot 43$ & 2618 & - & - & $\ldots$ & - & lait de vache \\
\hline 5508 & $1.12-13$ & 3481 & - & - & - & $\ldots$ & neuve. \\
\hline 5645 & $14-3-44$ & 3107 & - & - & - & - & \\
\hline 5546 & $30-1-44$ & 3000 & - & - & - & $\therefore$ & . \\
\hline 5643 & $15-3-44$ & 2666 & - & - & - & - & \\
\hline 5635 & $1-3-4.4$ & 2582 & 一 & - & $\therefore$ & - & \\
\hline 5582 & $1-2-44$ & 2762 & - & - & - & - & \\
\hline 5550 & $22-1-44$ & 3635 & - & - & - & réceptif & \\
\hline 5579 & $11-2 \cdot 44$ & 3500 & - & - & 一 & immun. & \\
\hline
\end{tabular}


Les expériences faites à Nioka sur la descendance de vaches immunisées à l'aide de virus peste bovine adapté sur chèrre, nous pormettent de faire les considérations suivantes :

$1^{0}$ L'influence immunisante du lait de mères vaccinées, pour les veaux à la mamelle, ne parait pas aussi importante qu'on pourrait le croire. Veaux $n^{\circ \mathbf{s}} 4889$, 4881, 4882.

20 Cette immunité empêchante constatée chez les veaux nés de mères vaccinées est bien une immunité congénitale, héritée de la mère, môme on l'abscnec de lait chargé d'anticorps pestiques (veaux $n^{\circ 5} 5736,5648$ ). La disparition de J'immunité chez le veau 5463 éprouvé rers l'àge de 7 mois est vraisemblablement due au temps écoulé entre la naissance et la date de-l'épreuve. Le veau 4895 s'est montré réceptif, quoique sa mère ait été vaccinée dix jours avant la mise bas. Celle-ci n'a pu donner à son produit une immunité qu'elle même ne possédait pas encore.

$3^{\circ}$ Sauf une exception (veau 5550 ), les veaur de mères-vaccinées mais compgns bien après la vaccination ont une immunité aussi marquée vis-à-vis du virus pestique adapté sur chèvre, que ceux nés de mères raccinées en état de gestation.

$4^{\circ} \mathrm{L}$ 'immunité congénitale des veaux vis-à-ris du virus peste boviue peut encore s'obsérver chez les veaux sevrés âgés de plus d'un an (veaux $n^{\circ s} 5048,5100$ ).

Il est probable que cette immunité congénitale est une des causes des variations observées dans l'expérimentation aree le virus adapté sur chèvre, par les divers chercheurs:

Opérant dans un milieu où la peste bovine n'est plus apparue depuis 1889, et sur des animaux nés de parents n'arant jamais fait la peste naturelle ou n'ayant jamais reçu de virus actif, nous avons pour 399 animaux inoculés et suivis journellement un seul animal qui n'a pas réagi. Ce dernier avait reçu une inoculation massive de virus pestique adapté sur chèrre, $100 \mathrm{cc}$. d'une émulsion de rate sèche à 1 pour 400 : température atteinte le matin $39{ }^{\circ} \mathrm{C}$, augmentation thermique $0,9^{\circ} \mathrm{C}$. A l'épreuve de l'immunité a vec virus bovin, cet animal s'est montré immun.

En opérant sur des animaux neufs, quelque soil leur âge, nous obtenons, avec le virus peste bovine adapté sur chèvre, pratiquement $100 \%$ de réagissants et d'animaux immuns. Pour ètre positive, la réaction de l'animal doit être caractérisée par une température de $39,5^{\circ} \mathrm{G}$ au moins, tempéralure prise Je matin avant 7 heures. Les veaux nés de mères activement immunisées ne peuvent ètre considérés comme neufs durant Ies premiers mois de la vie, certains mème jusqu'à plus d'un ar.

Nos pourcentages de réactions diflèrent de ceux de nos confrères anglais opérant dans l'Est Aflicain.

En Ouganda (2), F.R. Bexl obtient les pourcentages suivants à l'inoculation du seul virus adapté sur chère. A noter que les animaux en expérience ne comprennent aucun animal marqué $R$, donc immunisés antérieurement, et aucun reau à la mamelle.

$$
\begin{array}{rrr}
\text { Boma } & 1-71 \% \text { de réagissants } \\
2-75 \% & - \\
3-74 \% & - \\
4-100 \% & - \\
\text { Total ....76 \% } & -
\end{array}
$$


Par catégorie d'animaux, il obtient : taureaux, taurillons $85 \%$ réagissants

$\begin{array}{lll}\text { vaches } & 68 \% & - \\ \text { jeune bétail } & 69 \%\end{array}$

Au Kénya, des expériences faites au laboratoire de Kabete donnent pour le virus vaccin seul : 35 réagissants sur 35 animaux inoculés. En expérimentation en milieu indigène, pour 253 animaux inoculés, on obtient 191 réagissants seulement.

En conclusion : en cas de vaccination prophylactique et systématique des bovidés à l'aide du virus adapté sur chèvre (virus vaccin), il n'est pas à conseiller d'inoculer les veaux sevrés issus de mères immunisées activement (peste bovine naturelle, séro-infection, vaccino-infection, virus chèvre). Les jeunes animaux ayant reç le virus entre huit mois et un an doivent l'année suivante, soit entre un et deux ans, recevoir une nouvelle injection afin dimmuniser activement les animaux qui n'auraient pas réagi à la première inoculation du fait d'une immunité congénitale prolongée.

Nous avons parfois appelé le virus peste bovine adapté sur chèvre Virus Vaccin. Cette appellation est justifiée puisque le virus chèvre possède les propriétés d'un virus vaccin qui sont :

10 Une atténuation de la virulence. L'inoculation de ce virus par une voie quelconque et à doses excessives ne pruvuque pas chez l'animal injecté une affection mortelle et ne le transforme pas en un porteur de virus dangereux pour les animaux neufs.

$2^{\circ}$ Une valeur immunisante réelle.

Laboratoire vétérinaire de Nioka (juin 1944).

\section{BIBLIOGRAPHIE}

(1) H. Jacotot et $\mathrm{N}$. Colson. - Effet du vaccin contre la paste bovine chez les veaux issus de vaches activement immunisées. Bul. Soc. Path. Exct. T. 28, p. 74 .

(2) KVG experiment Teso. memo from Uganda : Protectorat. The Veterinary department, 6 octobre 1942.

(3) Report of proceeding of the second Conference on Rinderpest. Nairobi 1939, p. 39 et suivantes.

\section{A PROPOS DE L'IMMUNITÉ CONGENITALE DUE AU VIRUS PESTE BOVINE ADAPTE SUR CHEVRE} par J. GILLAIN

Une publication de juin 1944 (1), relative aux résultats obtenus à Nioka, suscite au $D^{r}$ R. DAubnEY, le distingué Directeur des Services vétérinaires au Kénya, les remarques suivantes :

" His results, obtained in the experiment on inherited immunity, are " much what I would have expected. If you note the age of the calves 
" when they were inoculated, you will find that with two exceptions "-No 5048 and $5100-$-all calves of seven months or over reacted to " the test, while all calves under the age of seven months failed to react " even including $N^{\text {To }} 5736$ and 5648, which had been fed, presumably, " on milk of suceptible cows. The results in the case of these two " animals suggest that calf immunity is as much, or possibly more, due " to age resistance, as to immunity transmitted by the milk. This I " think is a line worth following in a country where you have plenty " of certainly susceptible cows. You may find that a group of calves " born of completely susceptible mothers will give you exactly similar " results on test to the series from immune mothers that is included " in Grluarn's paper. This would be sufficient to establish that the " phenomenom is one of age resistance and not of inherited immunily. " It would be most valuable if Giclain could make such an observation " in Nioka."

Nous avons donc comparé la résistance des veaux nés de mères réceptives au virus Peste Bovine, à celle des veaux nés de mères vaccinées à l'aide du virus Peste Bovine adapté sur chèvre.

\section{TABLEAU No 1}

\begin{tabular}{|c|c|c|c|c|c|c|}
\hline $\begin{array}{c}\text { VEAU } \\
\text { No }\end{array}$ & NÊ LE & $\begin{array}{c}\text { No } \\
\text { DE Lid MÈRE }\end{array}$ & 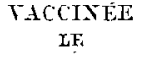 & $\begin{array}{l}\text { DATE } \\
\text { eprotlfe veail }\end{array}$ & RESBULTAIS & DBSFRYATIOXSS \\
\hline G 35 & $21-1-43$ & Sans No & Neuve & $10-5-43$ & Positif & - \\
\hline G 16 & $24-12-42$ & 1219 & $16-10-42$ & — & Négatif & 一 \\
\hline G 14 & $21-12-42$ & 1876 & Neuve & $\ldots$ & Positif & - \\
\hline G 37 & $12-2-43$ & Noire sans No & - & - & - & $\ldots$ \\
\hline G 10 & $10-12-42$ & Rouge sans $\mathrm{N}^{\circ}$ & - & — & -. & - \\
\hline G 18 & $4-1-43$ & Rouge pie & - & $14-5-43$ & - & Jumeaux \\
\hline G 19 & $4-1-43$ & - & & - & - & \\
\hline G 30 & $6-1-43$ & 1297 & $11-9-42$ & - & Négatif & - \\
\hline G 34 & $23-1-43$ & 01 & $30-10-42$ & - & Douteux & Voir T. 2 \\
\hline G 38 & $19-2-43$ & 1700 & 4.8 .42 & - & Négatif & - \\
\hline G 39 & $20-2-43$ & 1296 & $10-9-42$ & - & - & $\longrightarrow$ \\
\hline 5873 & $10-8-44$ & 3143 & Neuve & $18-1-40$ & Positif & 二 \\
\hline 5875 & $19-8-44$ & 3051 & - & — & — & - \\
\hline 5889 & $3-9-44$ & 1825 & - & - & - & - \\
\hline 5900 & $15-9-44$ & 2934 & - & - & - & - \\
\hline 5929 & $10-10-44$ & 2947 & - & 一 & - & - \\
\hline 5930 & $21-10-44$ & 3221 & 一 & - & - & 一 \\
\hline 5931 & $22-10-44$ & 2966 & - & - & 一 & - \\
\hline 5933 & $24-10-44$ & 3129 & $\ldots$ & - & 一 & - \\
\hline 5935 & $22-10-44$ & 3076 & $\ldots$ & - & - & - \\
\hline 6020 & $8-11-44$ & 3016 & - & - & - & - \\
\hline 6022 & $21-11-44$ & 3077 & - & 一 & - & 一 \\
\hline 5874 & $15-8-44$ & 3687 & $15-9-42$ & - & Négatif & - \\
\hline 5934 & $29-10-44$ & 3684 & - & - & - & — \\
\hline 6023 & $28-11-44$ & 3685 & $\ldots$ & - & - & $\ldots$ \\
\hline
\end{tabular}


TABLEAU No2

IMPORTANCE DES REACTIONS VACCINALES

\begin{tabular}{|c|c|c|c|c|c|c|c|}
\hline $\begin{array}{c}Y \operatorname{SeAU} \\
\text { No }\end{array}$ & M:ËRE & $\begin{array}{l}\text { DÉEUT } \\
\text { proussete thermique le }\end{array}$ & $\begin{array}{l}\text { MAXIMUM } \\
\text { BÁAOTION LE }\end{array}$ & $\begin{array}{l}\text { MAXTMUM } \\
\text { thelliqique alteilt }\end{array}$ & $\begin{array}{l}\text { Alfrullitilitor } \\
\text { thermique }\end{array}$ & \multicolumn{2}{|c|}{$\begin{array}{l}\text { DUREE } \\
\text { REACTYON }\end{array}$} \\
\hline G 33 & Neure & $2^{e}$ jour & $2^{e}$ jour & $40,4^{\circ} \mathrm{C}$ & $1,9^{\circ} \mathrm{C}$ & 11 & jours: \\
\hline G 16 & Vaccinée & \multicolumn{2}{|c|}{ pas de réaction } & & & & - \\
\hline G 14 & Neuve & $2^{\mathrm{e}}$ jour & $3^{\mathrm{e}}$ jour & $4.1,1$ & 2,5 & 6 & - \\
\hline G 37 & - & $2^{e}-$ & $3^{e}-$ & 41 & 2,5 & 10 & - \\
\hline G 10 & - & $3^{e}-$ & $4^{e}-1$ & 40,5 & 2,1 & 5 & - \\
\hline G 18 & - & $3 e-$ & $4^{e}-$ & 40,5 & 2,1 & 6 & - \\
\hline G. 19 & - & $3^{e}-$ & $5^{\mathrm{e}}-$ & 40,5 & 2 & 6 & - \\
\hline G 30 & Vaccinée & \multicolumn{2}{|c|}{ pas de réaction } & - & - & & 一 \\
\hline G 34 & - & $4^{\mathrm{e}}$ jour & $5^{\mathrm{e}}$ jour & 39,6 & 1 & 2 & - \\
\hline G 38 & — & \multirow{2}{*}{\multicolumn{2}{|c|}{$\begin{array}{l}\text { pas de réaction } \\
\text { pas de réaction }\end{array}$}} & - & - & & - \\
\hline G 39 & - & & & - & & & 一 \\
\hline 5873 & Neuve & $3^{\mathrm{e}}$ jour & 4. jour & 41,7 & 3,7 & 2 & - \\
\hline 5875 & - & $3^{e}$ & & 40,7 & 2,4 & 5 & - \\
\hline 5889 & - & $3^{\mathrm{e}}$ 一 & $4^{\mathrm{e}}-$ & 40,2 & 2,6 & 4 & - \\
\hline 5900 & 一 & - & $6^{\mathrm{e}}-$ & 39,8 & 1,8 & 1 & - \\
\hline 5929 & - & $3^{e}-$ & $3^{e}-$ & 40,3 & 2,1 & 3 & - \\
\hline 5 & & - & $3 \mathrm{e}-$ & 40 & 2,4 & 5 & - \\
\hline 31 & - & - & $4^{\mathrm{e}}-$ & 40,4 & 2,4 & 3 & - \\
\hline 5933 & $\longrightarrow$ & 40 & $\hat{5}^{\mathrm{e}}-$ & 39,7 & 2 & 3 & - \\
\hline & - & & $3^{e}-$ & 40,4 & 2,1 & 3 & - \\
\hline 6020 & _- & $3 \mathrm{e}-$ & $4^{\mathrm{e}}-$ & 40 & 2,2 & 3 & - \\
\hline 6022 & $\therefore-$ & $3^{e}-$ & $3^{\mathrm{e}}-$ & 40 & 2,2 & 4 & - \\
\hline $587 \overline{4}$ & Vaccinée & \multicolumn{2}{|c|}{ pas de réaction } & - & - & & - \\
\hline 59 & - & \multicolumn{2}{|c|}{ pas de réaction } & — & - & & - \\
\hline 6023 & - & \multicolumn{2}{|c|}{ I } & - & - & & 一 \\
\hline
\end{tabular}

Les veaux nés de mères neuves, donc réceptives au virus Peste Bovine, se montrent réceptifs, quel que soit leur âge. Le degré de réceptivité et de la réaction vaccinale des veaux nés de mères réceptives est comparable d̀ celui des bovidés plus âgés. Le 20 octobre 1942, l'injection du. virus adapté sur chèvre à 22 veaux à la mamelle nés de mères réceptives nous a donné, dans le mois de la vaccination, 7 mortalités dues à la réaction vaccinale (gastro-entérite, coccidiose).

Les veaux nés de mères vaccinées avec le virus Peste Bovine adapté sur chèvre accusent, entre 1 jour et 7 mois et parfois plus longtemps, une immunité très lorte.

Cette immunité existe tant pour les veaux nés des mères vaccinées durant la gestation que pour les veaux conçus bien après la vaccination de la mère. Il s'agit donc bien d'une immunité cong énitale et non d'une résistance particulière à l'âge des veaux.

Dans notre étude antérieure (2), nous avons montré que cette immunité n'est pas sous la dépendance de l'absorption de lait chargé d'anticorps pestiques.

Comme cette immunité est transitoire et disparait généralement à 
partir de l'àge de sept mois, il faut admettre qu'il s'agit simplement d'une i mmunité congénitale naturelle acquise de la mère. La vache donne cette immunité passive à son produit par le passage à travers le placenta de ses anticorps pestiques. L'immunité congénitale du produit ne peut donc exister que lorsque l'immunité est acquise par la mère. Si l'on prend comme moyenne la durée de plus ou moins quatorze jours nécessaire à l'établissement de l'immunité maternelle à partir de la vaccination, on comprend que les produits nés durant ces quatorze jours peuvent ne pas avoir d'immunité congénitale. C'est le cas du veau no 4895 (3) qui, né dix jours après la vaccination de la mère, ne montre aucune immunité.

Conclusion. - La résistance des jeunes reaux au virus pestique, veaux nés de mères vaccinées à l'aide du virus Peste Bovine adapté sur chèvre, est done bien une immunité congénitale et non une résistance nalurelle en relation avec l'àge des animaux.

Laboratoire vétérinaire de l'Inéac, à Nioka (février 1945).

Référence notes précédentes :

(1) Dr J. Gmutaix. - Immunité congénitale et virus Peste Bovine adapté sur chèvre. Juin 1944.

(2) $\mathrm{D}^{\mathrm{r}}$ J. Gillain. - Le virus Peste Borine adapté sur chèvre saponiné ou virus vaccin sapminé. Juillet 1944.

\section{EMPLOI DU VACCIN ANTIPESTIQUE FORMOLE ALUMINE : TEMPS NECESSAIRE POUR L'ETABLISSEMENT DE L'IMMUNITE par R. LARRAT, J. SULPICE, NIANG Sidy}

Nous avons voulu vérifier l'observation suivante, faite par plusieurs agents au cours des campagnes d'immunisation :

Dans un foyer récent, lorsqu'apparaît le premier cas et avant toute mortalité, l'emploi du vaccin formolé aluminé arrële généralement la marche de la contagion : l'établissement de l'immunité paraîl beaucoup plus rapide qu'avec le vaccin formolé ordinaire.

\section{Protocole :}

Des veaux supposés réceptifs, ayant sensiblement même taille et même poids ( 75 à $82 \mathrm{~kg}$ ), reçoivent, par groupes échelonnés, une dose de V.G.A. (2 cc.). Ils sont soumis, 3,5 ou 6 jours après la varcination, Elev. et Méd, vét. des Pays tropicaux. - Avril 1947. 